\title{
Tomato juice decreases LDL cholesterol levels and increases LDL resistance to oxidation
}

\author{
Marja-Leena Silaste ${ }^{1,2}$, Georg Alfthan ${ }^{3}$, Antti Aro $^{3}$, Y. Antero Kesäniemi ${ }^{1,2}$ and Sohvi Hörkkö ${ }^{2,4 *}$ \\ ${ }^{1}$ Department of Internal Medicine, University of Oulu, P.O. Box 5000, 90014 Oulu, Finland \\ ${ }^{2}$ Biocenter Oulu, P.O. Box 5000, 90014 University of Oulu, Oulu, Finland \\ ${ }^{3}$ National Public Health Institute (KTL), Mannerheimintie 166,00300 Helsinki, Finland \\ ${ }^{4}$ Department of Farmacology and Toxicology, University of Oulu, P.O. Box 5000, FIN-90014 Oulu, Finland
}

(Received 24 January 2007 - Revised 26 April 2007 - Accepted 30 April 2007)

High dietary intakes of tomato products are often associated with a reduced risk of CVD, but the atheroprotective mechanisms have not been established. This study was conducted to investigate the effects of increased dietary intake of tomato products on plasma lipids and LDL oxidation. The diet intervention included a baseline period, a 3-week low tomato diet (no tomato products allowed) and a 3-week high tomato diet (400 ml tomato juice and $30 \mathrm{mg}$ tomato ketchup daily). Twenty-one healthy study subjects participated in the study. Total cholesterol concentration was reduced by 5.9 (SD 10) \% (P=0.002) and LDL cholesterol concentration by 12.9 (SD 17.0) \% (P=0.0002) with the high tomato diet compared to the low tomato diet. The changes in total and LDL cholesterol concentrations correlated significantly with the changes in serum lycopene $(r 0.56$, $P=0.009 ; r 0.60, P=0.004$, total and LDL, respectively), $\beta$-carotene $(r 0.58, P=0.005 ; r 0.70, P<0.001)$ and $\gamma$-carotene concentrations $(r 0.64$, $P=0.002 ; r 0.64, P=0.002)$. The level of circulating LDL to resist formation of oxidized phospholipids increased $13 \%(P=0.02)$ in response to the high tomato diet. In conclusion, a high dietary intake of tomato products had atheroprotective effects, it significantly reduced LDL cholesterol levels, and increased LDL resistance to oxidation in healthy normocholesterolaemic adults. These atheroprotective features associated with changes in serum lycopene, $\beta$-carotene and $\gamma$-carotene levels.

Lycopene: Tomato: LDL: Cholesterol: Oxidation

Tomato is one of the most widely consumed fruit/vegetable in the Western world, and it has been estimated that, for example, in America the average annual consumption of fresh tomatoes is approximately $8 \mathrm{~kg}$ per person and processed tomato products is $31 \mathrm{~kg}$ per person, making tomatoes the most frequently consumed canned vegetable ${ }^{1}$. Many tomato products are good source of potassium, folate, and the vitamins $\mathrm{A}, \mathrm{C}$ and $\mathrm{E}$. In addition, tomatoes also contain valuable phytochemicals including carotenoids and polyphenols (such as $\alpha-, \beta-, \gamma$-carotene, lutein, lycopene, $\beta$-carotene) and flavonoids (USDA Nutrient Data Bank, http://www.bal. usda.gov). These tomato phytochemicals, especially lycopene, have been frequently associated with a reduced risk of $\mathrm{CVD}^{2-9}$

High dietary intakes of tomatoes and plasma concentrations of carotenoids have been associated with a reduced risk of $\mathrm{CVD}^{6,10,11}$. Recently, growing evidence from several epidemiological studies has indicated that lycopene, the major carotenoid in tomato, might be more important than other carotenoids in preventing atherosclerosis and $\mathrm{CVD}^{2-5,7,8}$. Lycopene is a fat-soluble pigment that gives tomatoes, guavas, pink grapefruit, watermelon and a limited number of other foods their red colour. Lycopene in tomatoes is released from cell wall protein-carotenoid complexes during processing, homogenizing and heating, and therefore, the bioavailability of lycopene is better from processed tomato products such as juice and sauce, for example, than from fresh tomatoes ${ }^{12,13}$. Lycopene is a powerful antioxidant in vitro, when the antioxidative capacity is measured as a capacity to quench singlet oxygen molecules ${ }^{14}$, and it has been speculated whether lycopene would be able to prevent the oxidative modification of LDL (oxidized LDL) in vivo. To date, the results from in vivo studies in man are not consistent: some studies have reported that dietary lycopene inhibits LDL oxidation ${ }^{15}$ whereas others have not observed such an effect $^{16}$. In addition to its powerful antioxidant properties, lycopene has been suggested to directly influence lipoprotein metabolism ${ }^{17,18}$.

The aim of the current study was to investigate the atheroprotective properties of increased daily dietary intake of tomato products on plasma lipids. We carried out an intervention, during which the dietary intake of tomato products increased by using tomato juice and ketchup, in addition to te habitual diets of the study subjects. We evaluated the influence of high versus low dietary tomato intake on serum lycopene, carotenoid and vitamin C levels, and on plasma total and LDL cholesterol levels, and on the capacity of LDL particles to resist oxidation. 


\section{Materials and methods}

\section{Subjects}

Twenty-one (five men and sixteen women) healthy, non-smoking subjects working at the University Hospital of Oulu volunteered for the study. The age, height and weight of the subjects ranged from 20 to 49 years (mean 30 years), from 152 to $187 \mathrm{~cm}$ (mean $169 \mathrm{~cm}$ ) and from 51 to $87 \mathrm{~kg}$ (mean $67 \mathrm{~kg}$ ), respectively. The average BMI of the subjects was 23.5 (SD 2.3$) \mathrm{kg} / \mathrm{m}^{2}$ at baseline, and it did not change during the study. The subjects had no medical treatments except for seven women who used oral contraceptives.

The study was carried out in accordance with the instructions of the Declaration of Helsinki. Informed consent was obtained from each participant. The intervention was approved by the Ethical Committee of the Faculty of Medicine, University of Oulu.

\section{Study design and diets}

The intervention consisted of a 2-week baseline period, 3 weeks of low tomato diet and 3 weeks of high tomato diet. The study subjects followed their habitual diets and eating patterns during the intervention. Before the intervention, the nutritionist interviewed all the participants and instructed them individually how to follow low and high tomato diets without changing their normal everyday habitual diets. In addition, the subjects received written instructions including a detailed list of all other known lycopene-containing foods. During the low tomato diet, the subjects were not to consume tomatoes, tomato products such as tomato ketchup, tomato sauces, tomato juice, etc., and other vegetables and fruit containing lycopene (watermelon, apricot, rosehip and guava). During the high tomato diet, the study subjects consumed $400 \mathrm{ml}$ tomato juice (Marli, Turku, Finland) containing $5.9 \mathrm{mg}$ lycopene $/ 100 \mathrm{ml}$ and $30 \mathrm{~g}$ tomato ketchup (Felix ${ }^{\circledR}$, Eslöv, Sweden) containing $12.4 \mathrm{mg}$ lycopene $/ 100 \mathrm{~g}$ daily, resulting in an average intake of $27 \mathrm{mg}$ dietary lycopene/d. The nutrient content of the tomato juice is presented in the Table 1. The participants were instructed to consume the tomato juice and ketchup with main meals (breakfast, lunch and dinner) during the day. The habitual consumption of tomatoes and other lycopene-rich foods was also allowed during the high tomato diet.

Table 1. The nutrient content of the tomato juice per $100 \mathrm{ml}$

\begin{tabular}{lc}
\hline Nutrient & Content \\
\hline Energy (kJ) & $87 \cdot 9$ \\
Energy (kcal) & 21 \\
Protein $(\mathrm{g})$ & $0 \cdot 6$ \\
Carbohydrate $(\mathrm{g})$ & $3 \cdot 8$ \\
Fat $(\mathrm{g})$ & $0 \cdot 3$ \\
Dietary fibre $(\mathrm{g})$ & $0 \cdot 4$ \\
Sodium $(\mathrm{mg})$ & $2 \cdot 5$ \\
Vitamin C $(\mathrm{mg})$ & 14 \\
Vitamin E $(\mathrm{mg})$ & $0 \cdot 7$ \\
Vitamin A $(\mu \mathrm{g})$ & 110 \\
Folic acid $(\mu \mathrm{g})$ & 11 \\
\hline
\end{tabular}

\section{Laboratory measurements}

Overnight fasting blood samples were drawn into EDTA-containing tubes for the measurement of plasma lipids, LDL oxidation, apoB, $\alpha$-carotene, $\beta$-carotene, $\gamma$-carotene, $\beta$-cryptoxanthin, lycopene and vitamin $\mathrm{C}$ at the baseline and at the end of both diet periods and the washout period. The plasma samples were kept at $-70^{\circ} \mathrm{C}$ until analysis.

\section{Measurements of plasma lipids}

To measure plasma lipids, plasma was separated by centrifugation at $1200 \mathrm{~g}(2600 \mathrm{rpm})$ for $15 \mathrm{~min}\left(4^{\circ} \mathrm{C}\right)$. The total plasma cholesterol and TAG concentrations were determined enzymatically using a Kone Spesific Analyzer (kits by Roche (Basel, Switerland); catalogue no. 114892320216 and catalogue no. 12016648 122). The HDL cholesterol concentration was determined after precipitation of the plasma sample with heparin-manganese chloride. LDL cholesterol was calculated by the Friedewald formula ${ }^{19}$.

\section{Measurement of LDL oxidation}

LDL oxidation was measured with a sandwich chemiluminescent immunoassay utilizing a well-characterized murine monoclonal antibody EO6 as previously described ${ }^{20,21}$. EO6 binds specifically to oxidized phospholipids in oxidized LDL. First, a polyclonal anti-human apoB-100 antibody (Biodesign (Saco, ME, USA); catalogue no. K45253G) was plated at $3 \mu \mathrm{g} / \mathrm{ml}$ overnight at $4^{\circ} \mathrm{C}$. After blocking with PBS buffer containing $1 \%$ bovine serum albumin, the plasma samples at 1:25 dilution (saturating concentration) were incubated for $1 \mathrm{~h}$ to capture LDL particles into the microtitre wells. The wells were washed three times with PBS to remove EDTA and subjected to oxidation by adding $\mathrm{CuSO}_{4}$ solution $(5 \mu \mathrm{M})$ and incubating at $37^{\circ} \mathrm{C}$ for $90 \mathrm{~min}$. The oxidation was stopped by washing the plates with EDTA containing PBS and the amount of oxidized phospholipid epitopes in apoB-100 particles was measured with biotinylated EO6. The antibody bound was detected with alkaline phosphatase-labelled Neutravidin (Pierce (Rockford, IL, USA); catalogue no. 31002 ) and LumiPhos 530 (Lumigen Inc., Southfield, MI, USA; catalogue no. P-501) substrate. Each plasma sample was measured in triplicate wells and all plasma samples from each study subject were in the same microtitre plate. In parallel wells the capture of equal amounts of apoB from the plasma samples was verified using biotinylated antiapoB antibody (Biodesign).

\section{Analysis of plasma carotenoids, $\alpha$-tocopherol and vitamin $C$}

The concentrations of $\alpha-, \beta-, \gamma$-carotenoids, lycopene and $\alpha$-tocopherol were determined by HPLC methods as described $^{22,23}$. Peak height/internal standard ratios were compared to the ratios of a secondary plasma standard whose values were traceable to National Institute of Standardization and Technology (Gaithersburg, MD, USA) certified serum materials, 968b. Lycopene isomers were resolved as two peaks, the first was designated trans isomers and the second cis isomers. The precision between series $(n 10)$ for $\beta$-cryptoxanthin, $\alpha$-carotene, $\beta$-carotene, $\gamma$-carotene, $\alpha$-tocopherol and 
trans and cis isomers of lycopene ranged between 5.4 and $7.9 \mathrm{CV} \%$.

For the measurement of plasma vitamin $\mathrm{C}, 0.5 \mathrm{ml}$ plasma was added to $4.5 \mathrm{ml} 5 \%$ metaphosphoric acid within $1 \mathrm{~h}$ after venepuncture and stored at $-70^{\circ} \mathrm{C}$. Total ascorbic acid was determined with an automated fluorimetric method using orthophenylenediamine and standardized against daily prepared ascorbic acid in $5 \%$ metaphosphoric acid ${ }^{24}$.

\section{Statistical methods}

The normality of distributions of all variables was first tested by the Shapiro-Wilk test. The distributions of plasma $\alpha$ - and $\beta$-carotene, and TAG were skewed, and their differences during the intervention were first tested by Friedman test for repeated measurements. The differences between the baseline period and diet periods were further tested by using the sign test. Student's $t$-test was used for variables that were normally distributed (plasma $\beta$-cryptoxanthin, $\gamma$-carotene, total lycopene, vitamin $\mathrm{C}$, total cholesterol, HDL cholesterol, LDL cholesterol and EO6 binding). Spearman's correlation coefficient was used to determine the associations between the variables that were skewed. The changes of the variables were normally distributed and Pearson correlation coefficient was used to test their associations. The differences were considered significant at the $5 \%$ level. The SPSS computer program version 9.0 (SPSS Inc., Chicago, IL, USA) was used in the statistical analyses. All values are expressed as mean and standard deviations, unless otherwise stated.

\section{Results}

\section{Plasma levels of carotenoids}

Fig. 1 demonstrates the serum levels of lycopene, $\alpha-, \beta$ - and $\gamma$ - carotenes, and $\alpha$-tocopherol levels during the different intervention diets. Plasma lycopene levels decreased significantly from the baseline $(0.307(\mathrm{SD} 0.149) \mathrm{mg} / \mathrm{l})$ in response to the low tomato diet $(0.130$ (SD 0.053$) \mathrm{mg} / \mathrm{l}, P(0.001)$, and increased significantly in response to the high tomato diet
(0.473 (SD 0.153$) \mathrm{mg} / \mathrm{l}, P(0 \cdot 001)$, as compared to both the baseline and the low tomato diet. Also, the plasma levels of $\beta$-carotene decreased significantly from the baseline $(0.280$ (SD 0.169$) \mathrm{mg} / \mathrm{l})$ in response to the low tomato diet $(0.240$ (SD 0.135$) \mathrm{mg} / \mathrm{l}, P=0.007$ ), and increased in response to the high tomato diet $(0.356$ (SD 0.151$) \mathrm{mg} / \mathrm{l}, P=0.007)$. Also, the plasma levels of $\gamma$-carotene decreased significantly from the baseline $(0.027$ (SD 0.012) $\mathrm{mg} / \mathrm{l})$ in response to the low tomato diet ( 0.015 (SD 0.0.006) $\mathrm{mg} / \mathrm{l}, P<0 \cdot 001)$, and increased in response to the high tomato diet $(0.066$ (SD 0.024) $\mathrm{mg} / \mathrm{l}$, $P<0.001)$. There were no statistically significant changes in the plasma levels of $\alpha$-carotene, $\beta$-cryptoxanthin or $\alpha$-tocopherol.

The plasma lycopene levels were related to $\gamma$-carotene levels at baseline $(r 0.84, P<0.001)$, on low tomato diet ( $r$ 0.67, $P=0.001)$ and on high tomato diet $(r \quad 0.89$, $P<0.001$ ) (data not shown). At baseline, plasma $\alpha$-carotene concentrations were related to plasma $\beta$-carotene levels $(r \quad 0.84, \quad P<0.001)$ and $\beta$-cryptoxanthin levels ( $r$ 0.81, $P<0.001)$ (data not shown). No other correlations between the plasma carotenoids were noticed.

\section{Plasma levels of total and LDL cholesterol}

Fig. 2(A) demonstrates that the plasma total cholesterol levels decreased significantly during the high tomato diet (4.19 (SD $0.78) \mathrm{mmol} / \mathrm{l}$ ), when compared to the baseline (4.43 (SD 0.64) $\mathrm{mmol} / \mathrm{l}, P=0.005)$ and to the low tomato diet (4.50 (SD 0.63) $\mathrm{mmol} / \mathrm{l}, \quad P=0.002)$. Also, the LDL cholesterol levels decreased significantly during the high tomato diet (2.18 (SD 0.62) $\mathrm{mmol} / \mathrm{l}$ ), when compared to the baseline $(2.44$ (SD 0.51 ) $\mathrm{mmol} / \mathrm{l}, P=0.002$ ) and to the low tomato diet (2.56 (SD 0.56) mmol/l, $P=0.0002$ ) (Fig. 2(B)). There were no significant differences in plasma total or LDL cholesterol levels between the baseline diet and the low tomato diet.

The average plasma total cholesterol concentration was reduced by 5.9 (SD 10) \% with the high tomato diet when compared to the low tomato diet, and 4.2 (SD 9.7) \% when compared to the baseline diet. The average LDL cholesterol concentration was reduced by 12.9 (SD 17.0) $\%$ with the

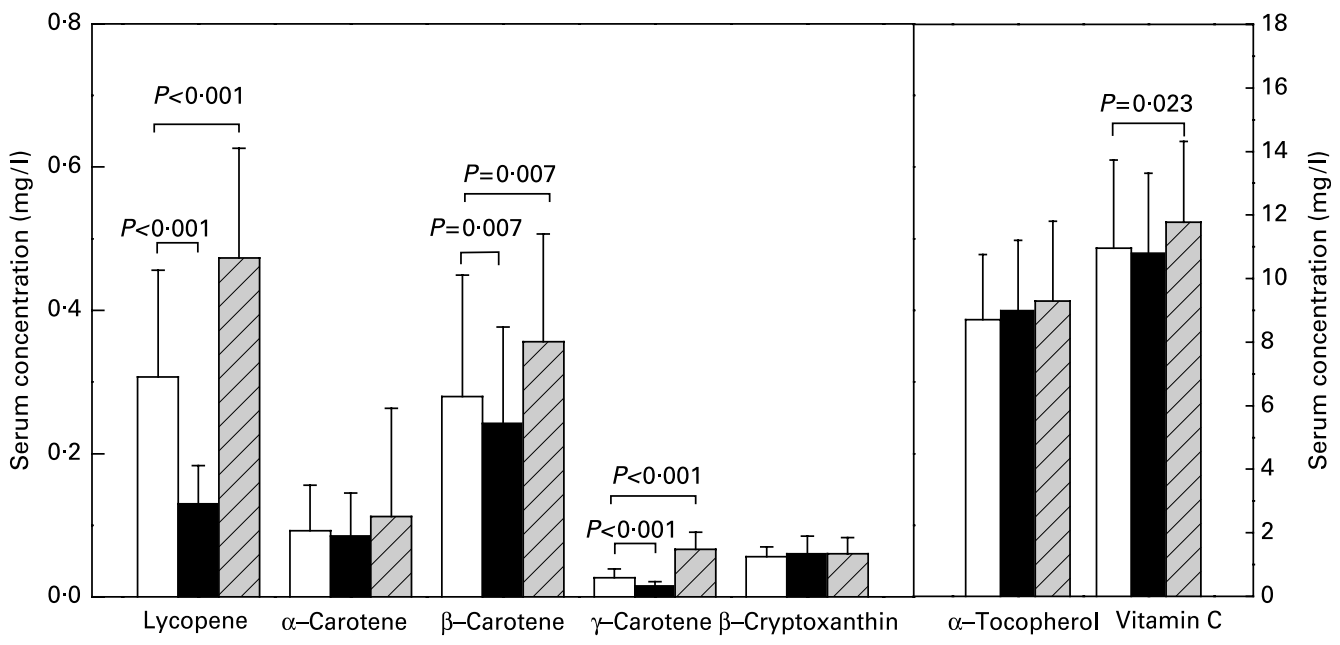

Fig. 1. The serum levels of lycopene, $\alpha-, \beta$ - and $\gamma$-carotenes, $\alpha$-tocopherol and vitamin $C$ during the baseline ( $\square$ ), the low tomato diet $(\square)$ and the high tomato diet (回).Values are means with standard deviations depicted by vertical bars $(n 21)$. 


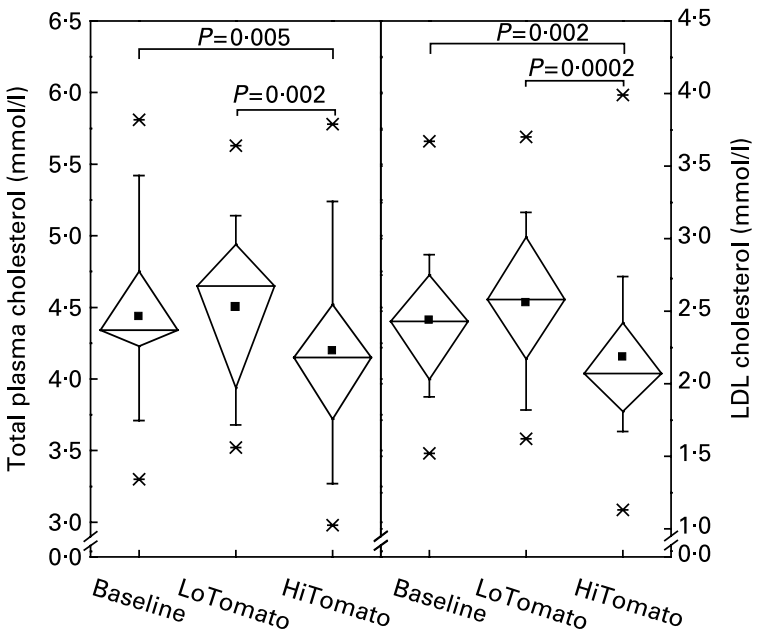

Fig. 2. The plasma total cholesterol levels (A) and LDL cholesterol levels (B) during the baseline, the low tomato diet (LoTomato) and the high tomato diet (HiTomato) ( $n$ 21). The box plots represent 25,50 and $75 \%$ and the vertical bars represent 10 and $90 \%$ distribution of the values; $*$, minimum and maximum values; $\mathbf{\square}$, mean values.

high tomato diet when compared to the low tomato diet, and 8.5 (SD 16.7) \% when compared to the baseline diet. The changes in plasma total and LDL cholesterol levels were not associated with the baseline plasma total or LDL cholesterol levels, i.e. subjects with high plasma total and LDL cholesterol levels responded similarly to those with low levels (data not shown). No significant changes were observed in the HDL cholesterol and TAG concentrations during the study.

\section{Associations between the changes in serum levels of} carotenoids and changes in plasma lipid levels

Fig. 3 demonstrates the associations between the absolute changes (as $\mathrm{mmol} / \mathrm{l}$ or $\mathrm{mg} / \mathrm{l}$ ) in plasma total cholesterol levels, LDL cholesterol levels and serum carotenoid levels in each of the study subjects between the high tomato diet $v$. the low tomato diet. Fig. 3 shows that the plasma total cholesterol levels were decreased in fifteen out of the twenty-one study subjects and LDL cholesterol levels were decreased in eighteen out of the twenty-one study subjects. The absolute changes in plasma total cholesterol concentrations correlated significantly with the absolute changes in serum lycopene $(r$ 0.56, $P=0.009$; Fig. 3(A)), $\beta$-carotene ( $r$ 0.58, $P=0.005$; Fig. 3(C)) and $\gamma$-carotene concentrations ( $r$ 0.64, $P=0.002 ;$ Fig. 3(E)). Similarly, the absolute changes in plasma LDL cholesterol concentrations associated significantly with the absolute changes in the serum lycopene ( $r$ 0.60, $P=0.004$; Fig. 3(B)), $\beta$-carotene $(r$ 0.70, $P<0.001$; Fig. 3(D) $)$ and $\gamma$-carotene concentrations $(r$ 0.64, $P=0.002$; Fig. 3(F)). These associations were positive, so that the study subjects with the highest increase in their serum carotenoid levels were the ones with the smallest decrease in their total or LDL cholesterol levels (Fig. 3). The changes in plasma total or LDL cholesterol levels were not associated with changes in $\alpha$-tocopherol levels (data not shown).

\section{Plasma levels of vitamin $C$}

The serum vitamin $\mathrm{C}$ levels increased significantly in response to the high tomato diet $(11.78$ (SD 2.53) $\mathrm{mg} / \mathrm{l}, P(0.05)$, when compared to the baseline diet (10.96 (SD 2.77) mg/l) (Fig. 1). No significant changes were observed in the vitamin $C$ levels between the baseline diet and the low tomato diet (Fig. 1). The absolute changes in serum vitamin $\mathrm{C}$ levels (as $\mathrm{mg} / \mathrm{l}$ ) associated significantly with the absolutes changes in serum lycopene levels $(r 0.53, P=0.01)$ and serum $\gamma$-carotene levels ( $r 0.61, P=0.003)$ between the high tomato diet and the low tomato diet among all the study subjects (Fig. 4).

\section{LDL oxidation}

The level of LDL oxidation was measured with a sensitive capture ELISA-method. In this method LDL particles are directly captured from plasma samples on to microtitre wells with an anti-apoB antibody and then subjected to oxidation with addition of copper ions. The oxidation is stopped and the amount of oxidation is measured with a murine monoclonal antibody EO6 specific for oxidized phospholipid epitope $^{20,25}$. Fig. 5 shows that LDL oxidation decreased significantly from the baseline (EO6 binding 107978 (SD 38478 ) relative light units (RLU)/ms) in response to the high tomato diet (EO6 binding 93220 (SD 40 732) RLU/ms) $(P=0.02)$. The EO6 binding was 13 (SD 36) \% lower on the high tomato diet compared to the baseline (Fig. 5). The amount of LDL oxidation was not associated with changes in plasma carotenoids or $\alpha$-tocopherol levels (data not shown).

\section{Discussion}

The major finding of the present study was that increased daily consumption of tomato juice and tomato tomato ketchup resulted in a significant decrease in LDL cholesterol concentration in combination with the ability of LDL particles to resist copper ion-induced oxidation in healthy normocholesterolaemic adults. Simultaneously, plasma levels of lycopene, $\beta$-carotene, $\gamma$-carotene and vitamin $\mathrm{C}$ levels increased.

The role of lycopene and other carotenoids abundant in tomato products in development and progression of atherosclerosis has been of vast interest among researchers in the past years (reviewed in Rao \& $\mathrm{Rao}^{26}$ ). Earlier epidemiological studies have suggested that lycopene may confer atheroprotection $^{2-8}$. Also, high adipose tissue lycopene levels have been shown to reduce the risk of developing a CVD by $48 \%{ }^{7}$. In contrast, low serum lycopene levels have been reported to associate with subclinical atherosclerosis, manifested as increased intima-media thickness of the common artery wall $^{4,5}$ and as calcified deposits in the abdominal aorta ${ }^{2}$. Furthermore, an inverse relationship has been demonstated between serum lycopene concentration and the risk of acute coronary events, such as myocardial infarction, and stroke ${ }^{3,6}$.

Despite the numerous epidemiological studies showing an inverse association between high consumption of tomato products and CVD, the protective mechanisms are scantily known. We found two possible mechanisms that may contribute to the atheroprotective effects of high intake of tomato products. First, just within 3 weeks of consuming the high amount of tomato products, the plasma total and LDL 

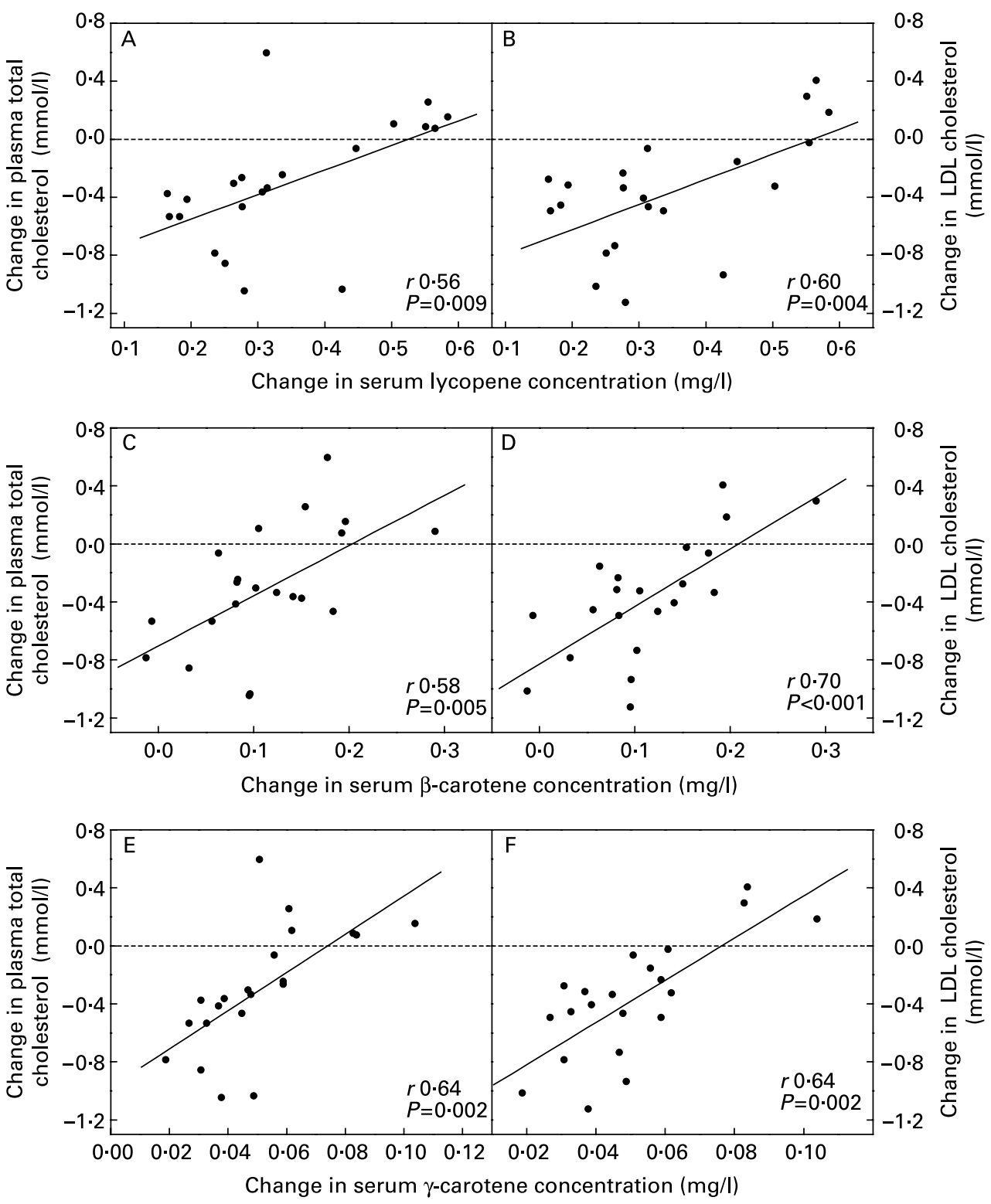

Fig. 3. The absolute changes in plasma total cholesterol levels (A, C, E) and plasma LDL cholesterol concentrations (B, D, F) in each individual study subject when comparing the high tomato diet to the low tomato diet. The changes in cholesterol levels are shown in relation to the changes in serum lycopene concentrations $(A, B)$, serum $\beta$-carotene concentrations $(C, D)$ and serum $\gamma$-carotene concentrations $(E, F)(n 21)$.

cholesterol levels dropped, by almost $6 \%$ and $13 \%$, respectively. These changes can be regarded as significant considering that the time period was only 3 weeks and that all of the study subjects were normocholesterolaemic at baseline. Moreover, no association was found between the baseline initial plasma cholesterol levels (which ranged from 3.30 to $5.81 \mathrm{mmol} / \mathrm{l}$ ) and the extent of the decrease in plasma cholesterol levels when comparing each individual subject in the high tomato diet $v$. the low tomato diet. The present data, although not conclusive, strongly suggest that subjects with 'higher' plasma cholesterol levels respond similarly to those with 'lower' plasma cholesterol levels. To date, only a few previous dietary interventions have tested the effect of tomato products or dietary lycopene on plasma cholesterol concentration in man. In another 3-week intervention study $^{27}$, a daily intake of $20 \mathrm{mg}$ lycopene from tomato juice or watermelon juice had no effect on the plasma lipid concentrations of ten subjects. In contrast, Hadley and co-workers found that a daily dose of $25-35 \mathrm{mg}$ lycopene from tomato soup or juice for 2 weeks resulted in a decrease of plasma total cholesterol levels from $5.23 \mathrm{mmol} / \mathrm{l}$ (baseline) to $4.75 \mathrm{mmol} / \mathrm{l}$ (intervention) ${ }^{17}$.

Several possible mechanisms for the decreased LDL cholesterol levels during the high tomato diet can be discussed. Previous cell cultures have shown that addition of lycopene to macrophages suppresses cellular cholesterol synthesis by approximately $40 \%$ by directly inhibiting cellular 3-hydroxy-3-methylglutaryl-CoA reductase activity ${ }^{18}$. This suppression was concomitant with increased uptake and degradation of LDL particles by the macrophages, suggesting 


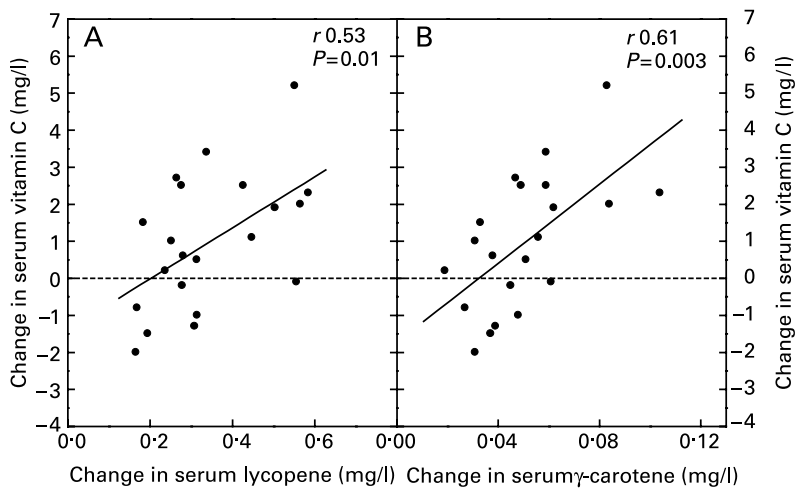

Fig. 4. The absolute changes (as $\mathrm{mg} / \mathrm{l}$ ) in serum vitamin $\mathrm{C}$ in relation to absolute changes in serum lycopene $(A)$ and $\gamma$-carotene $(B)$ concentrations, between the high tomato diet and the low tomato diet ( $n 21)$.

simultaneous up-regulation of LDL receptor activity ${ }^{18}$. Similar effects were also seen with $\beta$-carotene ${ }^{18}$. Finally, the same authors ${ }^{18}$ tested in vivo pure lycopene supplementation $(60 \mathrm{mg} / \mathrm{d})$ for 3 months in six healthy male subjects and showed that lycopene reduced plasma LDL cholesterol concentration by $14 \%$. In the present study, we observed a comparable reduction of $13 \%$ in the plasma LDL cholesterol concentration in response to a shorter period of intervention time (only 3 weeks) with tomato juice and ketchup. However, we were surprised that in the present study the correlations between the absolute changes in serum carotenoids (lycopene, $\beta$-carotene and $\gamma$-carotene) and plasma total and LDL cholesterol concentrations were positive. This means that

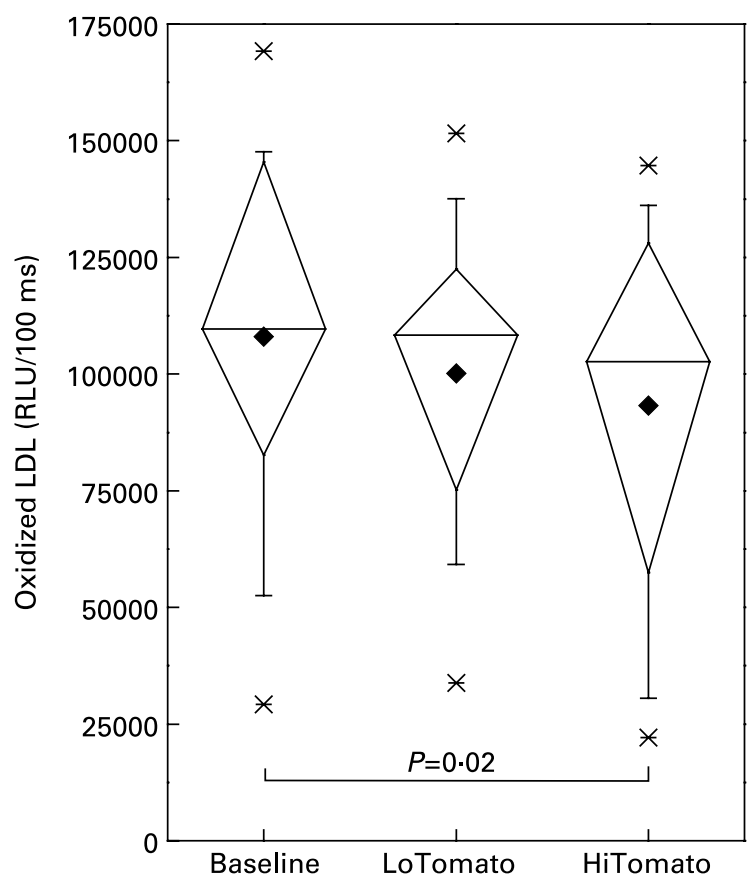

Fig. 5. The measurement of LDL oxidation during baseline, the low tomato diet (LoTomato) and high tomato diet (HiTomato) (n 21). The box plots represent 25,50 and $75 \%$ and the vertical bars represent 10 and $90 \%$ distribution of the values; $*$, minimum and maximum values; $\bullet$, mean values. RLU, relative light units. the more the serum lycopene (or $\beta$-carotene or $\gamma$-carotene) level increased from the low tomato diet to the high tomato diet, the less pronounced was the plasma total and LDL cholesterol decrement among all of the study subjects (as shown in Fig. 3). These correlations were marked, suggesting to us that the changes observed in total and LDL cholesterol concentrations were indeed related to the high intake of tomato products, but among the study subjects there is individual variability in response to the carotenoids. It may also be that there are some other components in the tomato product that affected cholesterol levels. Tomatoes are rich in other carotenoids, vitamins and flavonoids and these may also have accounted for the cholesterol-lowering effect. Tomatoes also contain steroidal glycoalkaloid tomatine, which is known to form strong insoluble 1:1 complexes with cholesterol molecules in vitro, and tomatine has been demonstrated to decrease plasma cholesterol levels in animals ${ }^{28,29}$.

The second possible mechanism contributing to the atheroprotective effects of high intake of tomato products that we found in the present study was the ability of the LDL particles to resist oxidation. In in vitro studies, lycopene has clearly demonstrated to be a more potent antioxidant than $\alpha$ - and $\beta$-carotene ${ }^{14}$, and the protective effect of lycopene has been primarily attributed to its antioxidant activity. The ability of lycopene to prevent oxidation of lipids in man has been tested in some previous diet interventions, but the results are conflicting. A number of previous studies have reported that increased dietary lycopene or consumption of tomato products reduces oxidative stress and oxidation of lipids ${ }^{15,17,30-34}$, whereas other investigators have not found any significant effect of lycopene supplementation on biomarkers of oxidative stress $^{16,27,35,36}$. We found that dietary supplementation with processed tomato products (tomato juice and ketchup) increased the resistance of LDL particles to oxidation. Methodologically, the present study is somewhat different to previous interventions. The ability of LDL to resist oxidation has been usually investigated by a measurement of lag phase of conjugated diene formation or formation of thiobarbituric acid reactive substances, when LDL isolated by sequential ultracentrifugation is exposed to copper ions ${ }^{16,17,34,36,37}$. In the present experiments, we captured the LDL particles directly from the plasma samples into microtitre wells with an anti-apoB antibody without ultracentrifugation. The LDL particles were subjected to copper-induced oxidation in the microtitre wells and the amount of oxidation was measured with a well-characterized monoclonal antibody EO6 which binds to oxidized phospholipid epitopes on the LDL particles ${ }^{20}$. This monoclonal antibody was cloned from apoE $^{-1-}$ mice and it binds to oxidized phospholipid containing the phosphorylcholine head group, but not to native unoxidized phospholipids, even though they contain the phosphorylcholine head group ${ }^{20,25,38}$. Oxidized phospholipids are a predominant component of oxidized LDL and have been demonstrated to have many pro-inflammatory and proatherogenic properties ${ }^{37}$. We found that the amount of oxidized LDL after oxidation, which represents the relative amount of oxidized phospholipids detected by EO6, was reduced by $13 \%$ in response to the high lycopene diet as compared to the baseline diet. Based on the findings of us and others $^{15,17,33,34}$, it seems that dietary lycopene may reduce 
lipid oxidation. However, we can only speculate that the decreased lipid oxidation seen in the present study was due to increase in plasma lycopene concentrations. Other carotenoids, vitamins, as well as flavonoids and related polyphenols, rich in tomato products may also have influenced the lipid peroxidation. For example, tomatoes contain flavanones, polyphenol derivatives, which have become well known in recent years for their powerful antioxidant potential (reviewed in Scalbert et $a l^{39}$ ). Polyphenols are plant-derived reducing agents conferring protection against LDL oxidation and oxidative stress, and may prevent various diseases, such as cancers, CVD and inflammation ${ }^{40-42}$. Interestingly, recent data suggest that some polyphenolic compounds possess lipid-lowering properties, by increasing faecal cholesterol excretion ${ }^{43}$, reducing intestinal cholesterol absorption $^{44}$ or perhaps by increasing cellular LDL receptor activity ${ }^{45}$.

In the present intervention, the lengths of the low and high tomato diets were 3 weeks. This can be considered sufficient, because the changes in the plasma levels of lycopene and other carotenoids occur rapidly with variation in dietary intake $^{17}$. Within these 3 weeks we were able to observe marked increase in serum concentrations of lycopene, and also $\beta$-carotene and $\gamma$-carotene in response to the high lycopene diet, which indicates that the compliance with the intervention was good. A potential limitation of the present intervention is that the low and high lycopene diets were not controlled and the dietary intake of the subjects was not monitored. However, the study subjects were highly motivated (researchers, doctors and other health professionals working in the university hospital) and they received specific information and instructions about the study diets from an experienced nutritionist, and they were carefully and individually advised how to follow the low and high tomato diets without changing their habitual eating patterns. Therefore, we are confident that the changes in the plasma total and LDL cholesterol concentrations and in the LDL oxidation did not result from changes in the habitual diets of the study subjects. In addition, the strong correlations between the changes of the plasma concentrations of cholesterol and carotenoids indicate that alterations in the consumption of tomato products contributed to the reduction of plasma cholesterol concentration. The effect of dietary lycopene on plasma lipid concentrations and on the oxidation of LDL should be confirmed in larger groups and in different populations, and also in subjects with higher initial plasma lipid levels.

In conclusion, we found that high daily dietary intake of tomato juice and ketchup significantly reduced LDL cholesterol levels in healthy normocholesterolaemic adults. Also, the ability of LDL particles to resist copper-induced formation of oxidized phospholipids increased. These atheroprotective features were well associated with the changes observed in serum lycopene, $\beta$-carotene and $\gamma$-carotene levels. The present study also raises a new question whether hypercholesterolaemic subjects would also benefit from the lipid-lowering properties of increased dietary intake of tomato products? Considering that tomato products are low in cost and rarely cause severe side-effects, the present data suggest that controlled large clinical and dietary interventions, as well as mechanistic studies, using tomato products are needed.

\section{Acknowledgements}

This work was supported by the Research Council for Health of the Academy of Finland, the Finnish Foundation for Cardiovascular Research, the Paulo Foundation and the Sigrid Juselius Foundation. The authors thank Ms Saija Kortetjärvi, Ms Liisa Mannermaa and Ms Sirpa Rannikko for their skilful laboratory assistance in this study.

\section{References}

1. Canene-Adams K, Campbell JK, Zaripheh S, Jeffery EH \& Erdman JW Jr (2005) The tomato as a functional food. J Nutr 135, 1226-1230.

2. Klipstein-Grobusch K, Launer LJ, Geleijnse JM, Boeing H, Hofman A \& Witteman JC (2000) Serum carotenoids and atherosclerosis. The Rotterdam Study. Atherosclerosis 148, 49-56.

3. Rissanen TH, Voutilainen S, Nyyssonen K, Salonen R \& Salonen JT (2001) Low serum lycopene concentration is associated with an excess incidence of acute coronary events and stroke: the Kuopio Ischaemic Heart Disease Risk Factor Study. Br J Nutr 85, 749-754.

4. Rissanen T, Voutilainen S, Nyyssonen K, Salonen R \& Salonen JT (2000) Low plasma lycopene concentration is associated with increased intima-media thickness of the carotid artery wall. Arterioscler Thromb Vasc Biol 20, 2677-2681.

5. Rissanen $\mathrm{TH}$, Voutilainen $\mathrm{S}$, Nyyssonen $\mathrm{K}$, Salonen R \& Salonen JT (2003) Serum lycopene concentrations and carotid atherosclerosis: the Kuopio Ischaemic Heart Disease Risk Factor Study. Am J Clin Nutr 77, 133-138.

6. Street DA, Comstock GW, Salkeld RM, Schuep W \& Klag MJ (1994) Serum antioxidants and myocardial infarction. Are low levels of carotenoids and alpha-tocopherol risk factors for myocardial infarction? Circulation 90, 1154-1161.

7. Kohlmeier L, Kark JD, Gomez-Gracia E, et al. (1997) Lycopene and myocardial infarction risk in the EURAMIC Study. Am J Epidemiol 146, 618-626.

8. Sesso HD, Buring JE, Norkus EP \& Gaziano JM (2004) Plasma lycopene, other carotenoids, and retinol and the risk of cardiovascular disease in women. Am J Clin Nutr 79, 47-53.

9. Sesso HD, Buring JE, Norkus EP \& Gaziano JM (2005) Plasma lycopene, other carotenoids, and retinol and the risk of cardiovascular disease in men. Am J Clin Nutr 81, 990-997.

10. Gaziano JM, Manson JE, Branch LG, Colditz GA, Willett WC \& Buring JE (1995) A prospective study of consumption of carotenoids in fruits and vegetables and decreased cardiovascular mortality in the elderly. Ann Epidemiol 5, 255-260.

11. Klipstein-Grobusch K, Geleijnse JM, den Breeijen JH, Boeing H, Hofman A, Grobbee DE \& Witteman JC (1999) Dietary antioxidants and risk of myocardial infarction in the elderly: the Rotterdam Study. Am J Clin Nutr 69, 261-266.

12. Gartner C, Stahl W \& Sies H (1997) Lycopene is more bioavailable from tomato paste than from fresh tomatoes. Am J Clin Nutr 66, 116-122.

13. van Het Hof KH, West CE, Weststrate JA \& Hautvast JG (2000) Dietary factors that affect the bioavailability of carotenoids. J Nutr 130, 503-506.

14. Di Mascio P, Kaiser S \& Sies H (1989) Lycopene as the most efficient biological carotenoid singlet oxygen quencher. Arch Biochem Biophys 274, 532-538.

15. Agarwal S \& Rao AV (1998) Tomato lycopene and low density lipoprotein oxidation: a human dietary intervention study. Lipids 33, 981-984.

16. Hininger IA, Meyer-Wenger A, Moser U, et al. (2001) No significant effects of lutein, lycopene or beta-carotene supplementation on biological markers of oxidative stress and LDL 
oxidizability in healthy adult subjects. $J$ Am Coll Nutr 20, 232-238.

17. Hadley CW, Clinton SK \& Schwartz SJ (2003) The consumption of processed tomato products enhances plasma lycopene concentrations in association with a reduced lipoprotein sensitivity to oxidative damage. $J$ Nutr $\mathbf{1 3 3}, 727-732$.

18. Fuhrman B, Elis A \& Aviram M (1997) Hypocholesterolemic effect of lycopene and beta-carotene is related to suppression of cholesterol synthesis and augmentation of LDL receptor activity in macrophages. Biochem Biophys Res Commun 233, 658-662.

19. Friedewald WT, Levy RI \& Fredrickson DS (1972) Estimation of the concentration of low-density lipoprotein cholesterol in plasma, without use of the preparative ultracentrifuge. Clin Chem 18, 499-502.

20. Hörkkö S, Bird DA, Miller E, et al. (1999) Monoclonal autoantibodies specific for oxidized phospholipids or oxidized phospholipid-protein adducts inhibit macrophage uptake of oxidized low-density lipoproteins. J Clin Invest 103, 117-128.

21. Silaste ML, Rantala M, Alfthan G, Aro A, Witztum JL, Kesäniemi YA \& Hörkkö S (2004) Changes in dietary fat intake alter plasma levels of oxidized low-density lipoprotein and lipoprotein(a). Arterioscler Thromb Vasc Biol 24, 498-503.

22. Bieri JG, Brown ED \& Smith JC (1985) Determination of individual carotenoids in human plasma by high performance liquid chromatography. J Liquid Chromatogr 8, 473-484.

23. Anttolainen M, Valsta LM, Alfthan G, Kleemola P, Salminen I \& Tamminen M (1996) Effect of extreme fish consumption on dietary and plasma antioxidant levels and fatty acid composition. Eur J Clin Nutr 50, 741-746.

24. Brubacher G \& Vuilleumier JP (1974) Vitamin C. In Clinical Biochemistry. Principles and Methods, 2nd ed., pp. 989-997 [HC Curtius and M Roth, editors]. Berlin: Walter de Gruyter.

25. Shaw PX, Hörkkö S, Chang MK, Curtiss LK, Palinski W, Silverman GJ \& Witztum JL (2000) Natural antibodies with the T15 idiotype may act in atherosclerosis, apoptotic clearance, and protective immunity. $J$ Clin Invest 105, 1731-1740.

26. Rao AV \& Rao LG (2007) Carotenoids and human health. Pharmacol Res 55, 207-216.

27. Collins J, Arjmandi B, Claypool P, Perkins-Veazie P, Baker R \& Clevidence B (2004) Lycopene from two food sources does not affect antioxidant or cholesterol status of middle-aged adults. Nutr $J \mathbf{3}, 15$.

28. Friedman M, Fitch TE \& Yokoyama WE (2000) Lowering of plasma LDL cholesterol in hamsters by the tomato glycoalkaloid tomatine. Food Chem Toxicol 38, 549-553.

29. Cayen MN (1971) Effect of dietary tomatine on cholesterol metabolism in the rat. J Lipid Res 12, 482-490.

30. Visioli F, Riso P, Grande S, Galli C \& Porrini M (2003) Protective activity of tomato products on in vivo markers of lipid oxidation. Eur J Nutr 42, 201-206.

31. Porrini M, Riso P, Brusamolino A, Berti C, Guarnieri S \& Visioli F (2005) Daily intake of a formulated tomato drink affects carotenoid plasma and lymphocyte concentrations and improves cellular antioxidant protection. Br J Nutr 93, 93-99.

32. Riso P, Visioli F, Grande S, Guarnieri S, Gardana C, Simonetti P \& Porrini M (2006) Effect of a tomato-based drink on markers of inflammation, immunomodulation, and oxidative stress. $J$ Agric Food Chem 54, 2563-2566.

33. Bub A, Watzl B, Abrahamse L, Delincee H, Adam S, Wever J, Muller H \& Rechkemmer G (2000) Moderate intervention with carotenoid-rich vegetable products reduces lipid peroxidation in men. J Nutr 130, 2200-2206.

34. Steinberg FM \& Chait A (1998) Antioxidant vitamin supplementation and lipid peroxidation in smokers. Am J Clin Nutr 68, 319-327.

35. Nelson JL, Bernstein PS, Schmidt MC, Von Tress MS \& Askew EW (2003) Dietary modification and moderate antioxidant supplementation differentially affect serum carotenoids, antioxidant levels and markers of oxidative stress in older humans. $J$ Nutr 133, 3117-3123.

36. Briviba K, Schnabele K, Rechkemmer G \& Bub A (2004) Supplementation of a diet low in carotenoids with tomato or carrot juice does not affect lipid peroxidation in plasma and feces of healthy men. J Nutr 134, 1081-1083.

37. Berliner JA, Subbanagounder G, Leitinger N, Watson AD \& Vora D (2001) Evidence for a role of phospholipid oxidation products in atherogenesis. Trends Cardiovasc Med 11, 142-147.

38. Binder CJ, Hörkkö S, Dewan A, et al. (2003) Pneumococcal vaccination decreases atherosclerotic lesion formation: molecular mimicry between Streptococcus pneumoniae and oxidized LDL. Nat Med 9, 736-743.

39. Scalbert A, Johnson IT \& Saltmarsh M (2005) Polyphenols: antioxidants and beyond. Am J Clin Nutr 81, 215S-217S.

40. Hayek T, Fuhrman B, Vaya J, Rosenblat M, Belinky P, Coleman R, Elis A \& Aviram M (1997) Reduced progression of atherosclerosis in apolipoprotein E-deficient mice following consumption of red wine, or its polyphenols quercetin or catechin, is associated with reduced susceptibility of LDL to oxidation and aggregation. Arterioscler Thromb Vasc Biol 17, $2744-2752$.

41. Manach C, Mazur A \& Scalbert A (2005) Polyphenols and prevention of cardiovascular diseases. Curr Opin Lipidol 16, $77-84$.

42. Scalbert A, Manach C, Morand C, Remesy C \& Jimenez L (2005) Dietary polyphenols and the prevention of diseases. Crit Rev Food Sci Nutr 45, 287-306.

43. Yokozawa T, Nakagawa T \& Kitani K (2002) Antioxidative activity of green tea polyphenol in cholesterol-fed rats. J Agric Food Chem 50, 3549-3552.

44. Ikeda I, Imasato Y, Sasaki E, Nakayama M, Nagao H, Takeo T, Yayabe F \& Sugano M (1992) Tea catechins decrease micellar solubility and intestinal absorption of cholesterol in rats. Biochim Biophys Acta 1127, 141-146.

45. Davalos A, Fernandez-Hernando C, Cerrato F, et al. (2006) Red grape juice polyphenols alter cholesterol homeostasis and increase LDL-receptor activity in human cells in vitro. $J$ Nutr 136, 1766-1773. 